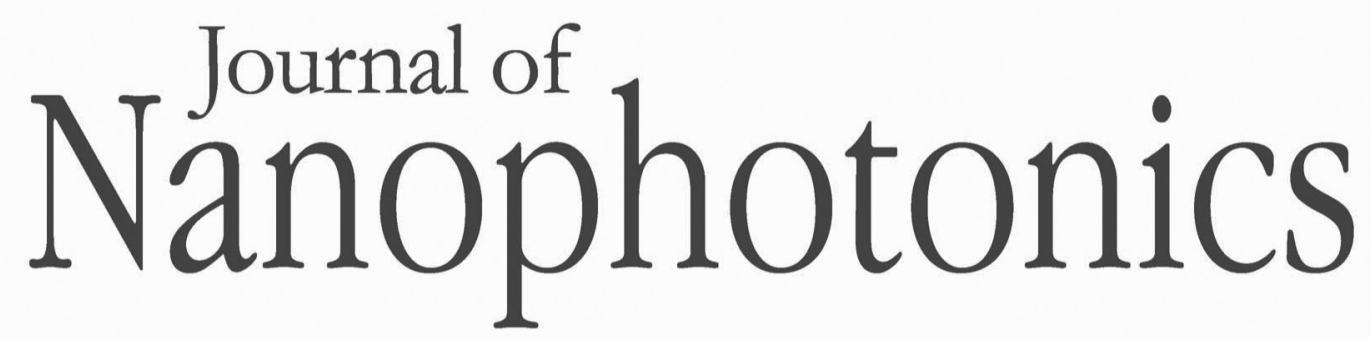

SPIEDigitalLibrary.org/jnp

\title{
Light management through guided- mode resonances in thin-film silicon solar cells
}

Tanzina Khaleque

Robert Magnusson 


\title{
Light management through guided-mode resonances in thin-film silicon solar cells
}

\author{
Tanzina Khaleque and Robert Magnusson* \\ University of Texas at Arlington, Department of Electrical Engineering, P.O. Box 19016, \\ Arlington, Texas 76019
}

\begin{abstract}
We theoretically explain and experimentally demonstrate light trapping in thin-film solar cells through guided-mode resonance (GMR) effects. Resonant field enhancement and propagation path elongation lead to enhanced solar absorption. We fabricate nanopatterned solar cells containing embedded $300-\mathrm{nm}$ period, one-dimensional gratings. The grating pattern is fabricated on a glass substrate using laser interference lithography followed by a transparent conducting oxide coating as a top contact. A 320-nm thick p-i-n hydrogenated amorphous silicon solar cell is deposited over the patterned substrate followed by bottom contact deposition. We measure optical and electrical properties of the resonant solar cells. Compared to a planar reference solar cell, around 35\% integrated absorption enhancement is observed over the 450 to 750-nm wavelength range. This light-management method results in enhanced short-circuit current density of $14.8 \mathrm{~mA} / \mathrm{cm}^{2}$, which is a $\sim 40 \%$ improvement over planar solar cells. Our experimental demonstration proves the potential of simple and well-designed GMR features in thinfilm solar cells. (C) The Authors. Published by SPIE under a Creative Commons Attribution 3.0 Unported License. Distribution or reproduction of this work in whole or in part requires full attribution of the original publication, including its DOI. [DOI: 10.1117/1.JNP.8.083995]
\end{abstract}

Keywords: guided-mode resonance; interferometric lithography; light trapping; thin-film solar cells.

Paper 13139SS received Dec. 13, 2013; revised manuscript received Jan. 24, 2014; accepted for publication Feb. 6, 2014; published online Mar. 6, 2014.

\section{Introduction}

Thin-film solar cell technology offers benefits of low-cost material usage and processing relative to currently dominant crystalline silicon solar cells. In contrast to classic thick, wafer-based silicon cells, the higher absorption coefficients of materials used in thin-film photovoltaics allow for a film thickness of hundreds of nanometers to micrometers. The quality of material can be relatively poor since the charge carriers only travel a distance on the order of the film thickness. However, the low-energy photons suffer from short optical paths that ultimately cause low spectral uptake in the cells near the material's bandedge. ${ }^{1-3}$ Consequently, efficient light-trapping mechanisms are necessary to obtain comparable performance from thin-film solar cells.

By incorporating properly designed photonic nanostructures, incoming sunlight can be trapped inside the absorbing layer while reducing loss caused by reflection and scattering. With a highly concentrated field, a thin absorbing layer sufficiently absorbs most of the solar spectrum. This allows further reduction of the absorbing layer thickness and ensures minimal usage of materials and better collection efficiency for low-quality materials. Numerous studies have been conducted to improve light-capture and collection efficiency of thin absorbing layers. The application of diffractive optics, ${ }^{4-6}$ random texturing, ${ }^{7,8}$ antireflective layers, ${ }^{9,10}$ plasmonics, ${ }^{11-13}$ photonic crystals, ${ }^{14-16}$ guided-mode excitation, ${ }^{6,17,18}$ and three-dimensional structures like nanowire, nanodome, and nanocone solar cells ${ }^{19-21}$ shows distinguished improvements in solar absorption. Though each mechanism contributes to the manipulation of optical path lengths inside the films, the most efficient light-harvesting scheme is yet to be convincingly identified. Using numerical simulation tools, it is possible to design

*Address all correspondence to: Robert Magnusson, E-mail: magnusson@uta.edu 
microdomain or nanodomain photonic structures showing enhanced absorption and photocurrents. However, from a practical point of view, application of such features into solar cells has to be technologically and economically feasible.

Here, we report guided-mode resonance (GMR) effects in waveguide-grating structures for enhanced optical absorption. We experimentally demonstrate the application of simple designs of one-dimensional (1-D) nanograting patterns into thin-film hydrogenated amorphous silicon (a-Si:H) solar cells. With these particular operative effects, the periodic spatial pattern couples the input sunlight into a collection of waveguide modes associated with thin wave-guiding films that resonate in the cell and concentrate the light in the active layer. Thereby, the photon interaction path is elongated within the silicon film, which helps particularly to harvest the low-energy photons. We describe the fabrication processes and present experimental results that comply with our theoretical analysis.

\section{GMR Effect in Lossy Layers}

A subwavelength periodic pattern and a waveguide layer over a substrate constitute a generic GMR element, as shown in Fig. 1(a), with characteristic parameters, period $\Lambda$, grating depth $d_{\mathrm{g}}$, waveguide layer thickness $d_{\mathrm{wg}}$, fill factor $F$, incident beam $I$, reflected beam $R$, and transmitted beam $T$. At resonance, an incident light beam couples to leaky waveguide modes through the grating structure. An efficient energy exchange occurs between the reflected and transmitted waves, resulting in sharp peaks in the diffraction efficiency spectrum of the resonant waveguide grating. ${ }^{22-24}$

The grating couples the incident wave into the resonant waveguide-grating structure. A resonance occurs when one of the diffracted waves generated by the grating element phase matches to a leaky waveguide mode admitted by the waveguide structure, as schematically depicted in Fig. 1(b). At the phase-matching condition, the effective mode propagation constant $\beta_{i}$, corresponding to the $i$ 'th order evanescent diffracted wave, is given by ${ }^{24}$

$$
\beta_{i}=k_{0}\left(n_{\mathrm{c}} \sin \theta_{\text {in }}-i \lambda / \Lambda\right),
$$

where $k_{0}=2 \pi / \lambda, \lambda$ is the free space wavelength, $n_{\mathrm{c}}$ is the refractive index of cover medium, $\theta_{\text {in }}$ is the incident angle, and $\Lambda$ is the period of the grating. The refractive index of the waveguide $\left(n_{\mathrm{wg}}\right)$ should be such that $n_{\mathrm{wg}}>n_{\mathrm{c}}, n_{\mathrm{s}}, n_{\mathrm{g}}$, where $n_{\mathrm{s}}$ is the refractive index of substrate and $n_{\mathrm{g}}$ is the average refractive index of the grating layer.

Depending on the device parameters, operating wavelength, and incident angle, there can be a single resonance or multiple resonances. Using powerful electromagnetic design methods, the spectral bands of these resonant leaky-mode elements can be engineered for various photonic

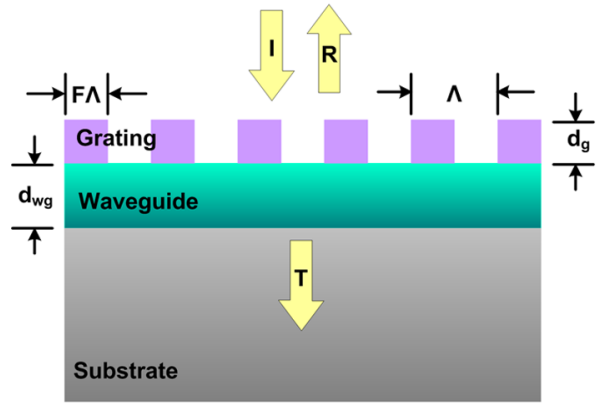

(a)

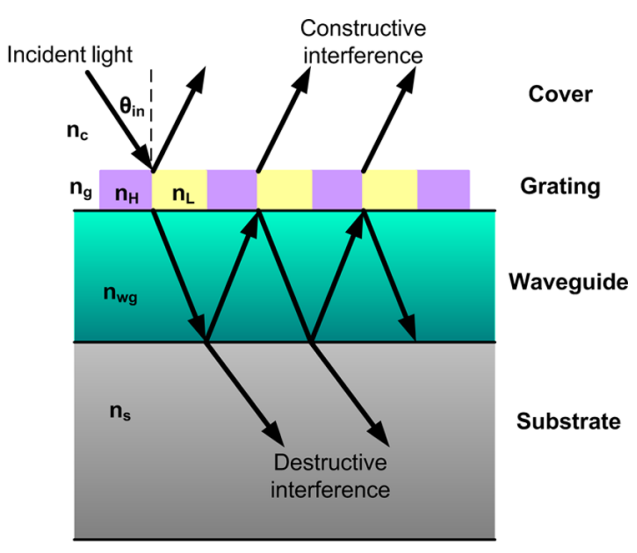

(b)

Fig. 1 (a) Schematic view of a guided-mode resonance (GMR) element featuring period $\Lambda$, grating depth $d_{\mathrm{g}}$, waveguide thickness $d_{\mathrm{wg}}$, and fill factor $F$. (b) Schematic GMR coupling at resonance according to the model provided by Rosenblatt et al. ${ }^{25}$ 


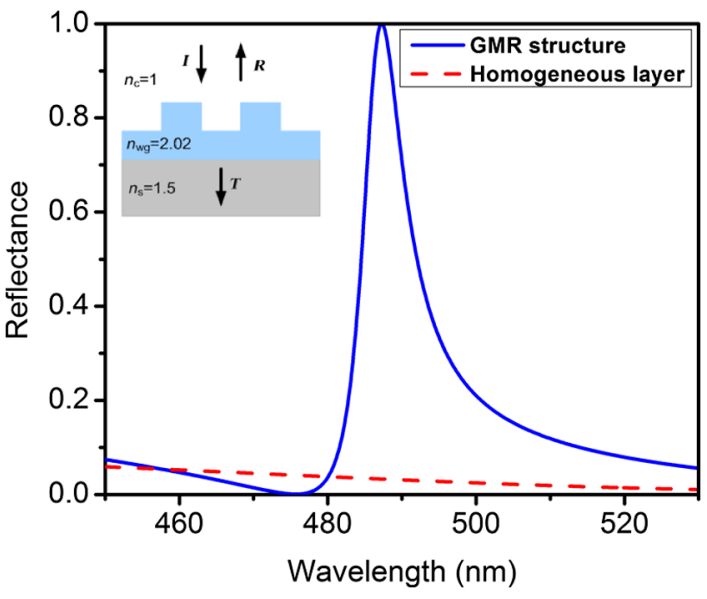

Fig. 2 GMR spectral response of a waveguide-grating structure with $\Lambda=280 \mathrm{~nm}, d_{\mathrm{g}}=55 \mathrm{~nm}$, $d_{\mathrm{wg}}=110 \mathrm{~nm}, F=0.5, n_{\mathrm{wg}}=n_{\mathrm{H}}=2.02, n_{\mathrm{c}}=n_{\mathrm{L}}=1$, and $n_{\mathrm{s}}=1.5$. The GMR occurs at the 487-nm wavelength with transverse electric (TE) polarized normally incident light. The dashed line represents the reflectance of a corresponding homogeneous layer with the grating replaced by a simple layer with an effective refractive index.

device applications. For example, it has already been shown that a single periodic layer with 1-D periodicity enables narrow line filters, polarizers, reflectors, and polarization-independent elements. ${ }^{26-28}$ An example of spectral response of a single-resonance device is shown in Fig. 2. For the computations, we use a computer code based on rigorous coupled-wave analysis (RCWA), ${ }^{29}$ which is a proficient algorithm to evaluate diffraction efficiency of periodic structures as further discussed in Ref. 30. In our numerical simulations, we use 11 harmonics and we verify good convergence by test simulations using 21 harmonics. For a simple waveguide-grating structure ${ }^{28}$ with $\Lambda=280 \mathrm{~nm}, d_{\mathrm{g}}=55 \mathrm{~nm}, d_{\mathrm{wg}}=110 \mathrm{~nm}, F=0.5, n_{\mathrm{wg}}=n_{\mathrm{H}}=2.02, n_{\mathrm{c}}=n_{\mathrm{L}}=1$, and $n_{\mathrm{s}}=1.5$, a complete energy exchange occurs between transmitted and reflected waves and a sharp resonance reflectance peak occurs at the 487-nm wavelength when illuminated with normally incident transverse electric (TE: electric field vector being orthogonal to the plane of incidence) polarized light. On the contrary, a homogeneous layer with an effective refractive index of the grating layer does not exhibit such spectral signature, as evidenced by dashed lines in Fig. 2.

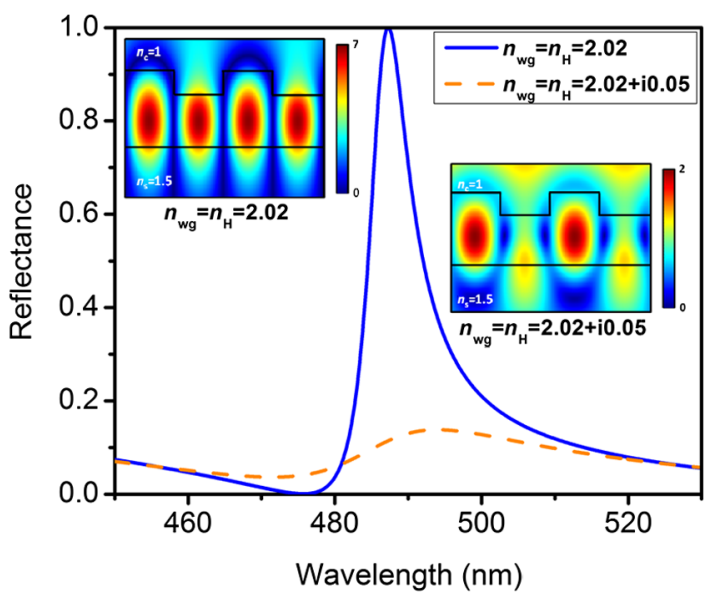

Fig. 3 GMR spectral response of a waveguide-grating structure with $\Lambda=280 \mathrm{~nm}, d_{\mathrm{g}}=55 \mathrm{~nm}$, $d_{\mathrm{wg}}=110 \mathrm{~nm}, F=0.5, n_{\mathrm{c}}=n_{\mathrm{L}}=1$, and $n_{\mathrm{s}}=1.5$. The solid line represents the GMR effect for lossless material where $n_{\mathrm{wg}}=n_{\mathrm{H}}=2.02$, and the dashed line represents the reduced reflectance for lossy material where $n_{\mathrm{wg}}=n_{\mathrm{H}}=2.02+\mathrm{i} 0.05$. The total internal electric field distributions for both lossless and lossy materials at the resonance wavelength of $487 \mathrm{~nm}$ are shown as insets. 


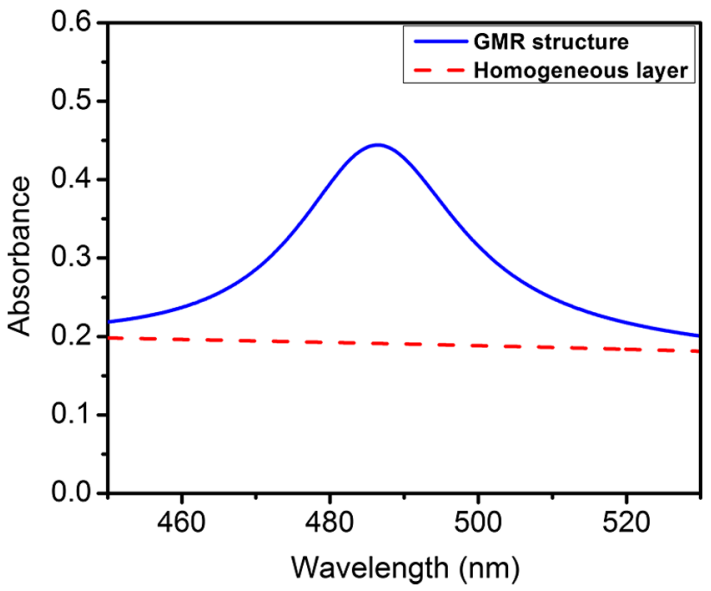

Fig. 4 The solid line represents TE polarized absorbance spectra of a GMR structure with $\Lambda=280 \mathrm{~nm}, d_{\mathrm{g}}=55 \mathrm{~nm}, d_{\mathrm{wg}}=110 \mathrm{~nm}, F=0.5, n_{\mathrm{c}}=n_{\mathrm{L}}=1, n_{\mathrm{s}}=1.5$, and $n_{\mathrm{wg}}=n_{\mathrm{H}}=$ $2.02+$ i0.05. The dashed line represents the absorbance of a corresponding homogeneous layer with $\kappa=0.05$. The absorbance is 2.3 times greater for the GMR element at the resonance wavelength of $487 \mathrm{~nm}$.

However, the GMR effect is strongly sensitive to material loss. The presence of material loss (complex refractive index $N=n+\mathrm{i} \kappa$, where $\kappa$ is the extinction coefficient) diminishes the external signatures of the resonance. If $\kappa=0.05$ is considered in the previous example $\left(n_{\mathrm{wg}}=n_{\mathrm{H}}=2.02+\mathrm{i} 0.05\right)$, the reflectance decreases significantly, as depicted in Fig. 3 . The total internal electric field distributions of these elements for both lossless and lossy materials are shown as insets in Fig. 3. We observe that even though the reflectance decreases, the field distribution with loss maintains key features with lower field levels. This shows that the fieldenhancement aspect of the GMR effect can be beneficial without the traditional external signatures being present. If we compare the absorbance with a planar reference, an enhancement ratio of around 2.3 is obtained at the resonance wavelength, as shown in Fig. 4.

\section{GMR-Enabled Light Management}

In this study, our interest lies in showing absorption enhancement in thin-film solar cells by applying the GMR effect. The grating layer, which is a phase-matching element, forces the incoming light into multiple resonances in the device, eventually increasing the probability of light absorption. A simple planar structure fails to offer such light confinement. Thus for efficient solar absorption, the GMR structure should support multiple resonances over the broadband solar spectrum. Designing the grating-pattern period to be smaller than the wavelengths in the solar spectrum $(\Lambda<\lambda)$ allows the GMR structure to operate as multiple resonant devices. To demonstrate the multiresonant GMR spectral response, we consider a structure consisting of an a-Si:H grating and a waveguide layer over a glass substrate with $\Lambda=350 \mathrm{~nm}, d_{\mathrm{g}}=50 \mathrm{~nm}$, $d_{\mathrm{wg}}=250 \mathrm{~nm}$, and $F=0.5$. Hydrogenated a-Si has a complex dispersive refractive index, $N(\lambda)=n(\lambda)+\mathrm{i} \kappa(\lambda)$, capable of absorbing light up to the 750-nm wavelength corresponding to bandgap energy of $1.7 \mathrm{eV}$. The numerical value of $N(\lambda)$ provided by Fontcuberta $\mathrm{i}$ Morral and Roca i Cabarrocas ${ }^{31}$ is given in Fig. 5.

Figure 6 shows the spectral responses of a multiresonant GMR structure with three different $\kappa$ values. Plot 1 indicates lossless a-Si:H with $\kappa=0$, plot 2 indicates a constant $\kappa=0.05$, and plot 3 indicates the original dispersive $\kappa(\lambda)$ over the spectrum, while $n(\lambda)$ remains the same (as in Fig. 5) for all three plots. The observed resonance peaks originate from input light coupling to leaky-modes across the spectrum. We observe the reduction of reflection and subsequent enhancement of absorption with the increase of the $\kappa$ value over the spectrum of 450 - to 750-nm wavelengths.

The total electric field patterns for the three different $\kappa$ values $(\kappa=0, \kappa=0.05$, and $\kappa=0.082)$ at the 651-nm resonance wavelength are shown in Fig. 7. The original value of $\kappa$ at $651 \mathrm{~nm}$ is 0.082. 


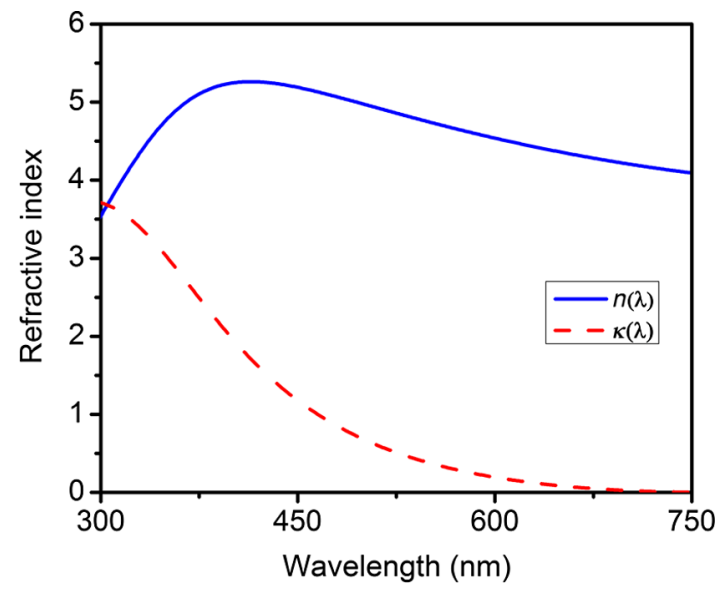

Fig. 5 Dispersive refractive index of a-Si:H as derived from Ref. 31.

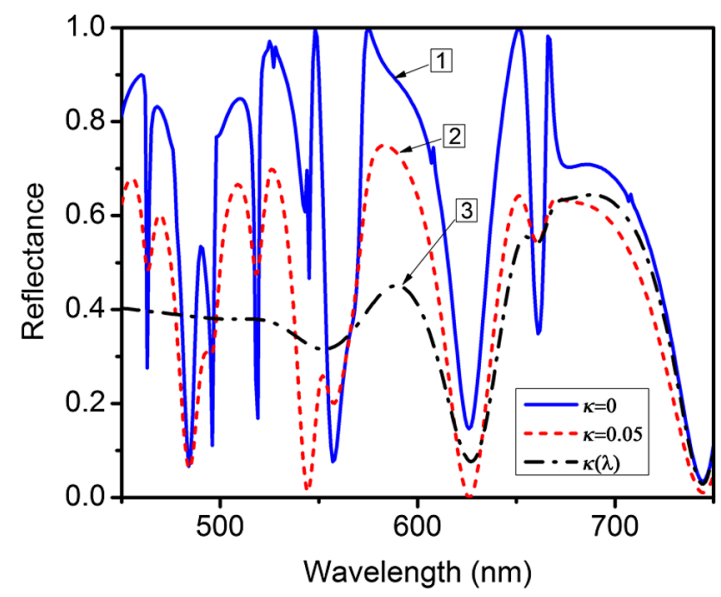

Fig. 6 GMR spectral response for a waveguide-grating structure with $\Lambda=350 \mathrm{~nm}, d_{\mathrm{g}}=50 \mathrm{~nm}$, $d_{\mathrm{wg}}=250 \mathrm{~nm}, F=0.5, n_{\mathrm{c}}=n_{\mathrm{L}}=1$, and $n_{\mathrm{s}}=1.5$. Plot 1 represents the GMR effect for lossless material where $\kappa=0$, plot 2 represents the reduced reflectance for lossy material where $\kappa=0.05$, and plot 3 represents reflectance for lossy a-Si:H with the original dispersive $\kappa(\lambda)$.
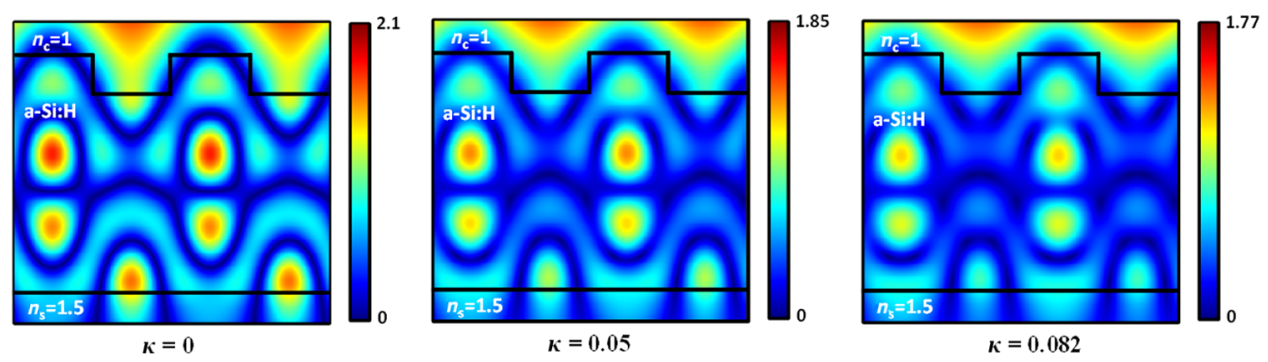

Fig. 7 Total electric field distribution inside an a-Si:H waveguide-grating structure for three different values of $\kappa(0,0.05$, and 0.082$)$ at $\lambda=651 \mathrm{~nm}$. The original $\kappa$ value of a-Si:H at $651 \mathrm{~nm}$ is 0.082 .

The field pattern gradually decays with the increase of the $\kappa$ value. Based on the simulation results, we intend to implement the photonic resonant structure into a real p-i-n thin-film a-Si:H solar cell to observe enhanced absorption through intensified field patterns and subsequent increased electrical efficiency. However, fabrication of resonant patterns into the active region of a planar solar cell requires sacrifice of materials and junction interruption, which will cause electrical performance degradation. Instead, we consider a superstrate cell configuration to incorporate the 


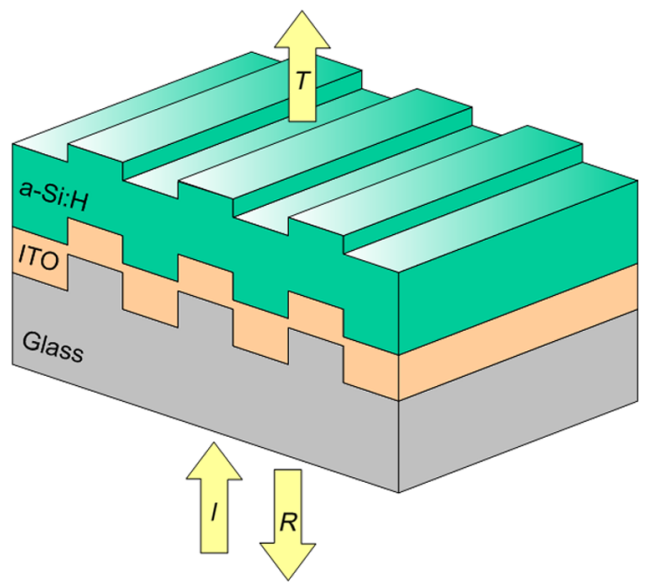

Fig. 8 Schematic view of a GMR solar cell without bottom contact.

patterns into the active region as schematically shown in Fig. 8. We fabricate a nanopattern on a glass substrate, and the pattern is gradually transferred to the silicon region without interrupting the junctions. We consider a thin indium-tin-oxide (ITO) layer in between the glass and silicon as a top contact. To observe the GMR-induced absorbance enhancement exclusively, we conduct the optical characterization before the bottom contact is made.

\section{Fabrication}

We fabricate the 1-D nanograting patterns on a glass substrate; Fig. 9 summarizes the fabrication steps. First, a $1 \times 1$ in. $^{2}$ glass substrate is cleaned with acetone, isopropanol, and deionized water and dried with blown nitrogen. Then an 80-nm bottom antireflection coating (BARC: DUV30J6) is spin-coated over the glass substrate at $1200 \mathrm{rpm}$ and baked for $60 \mathrm{~s}$ on a heating plate

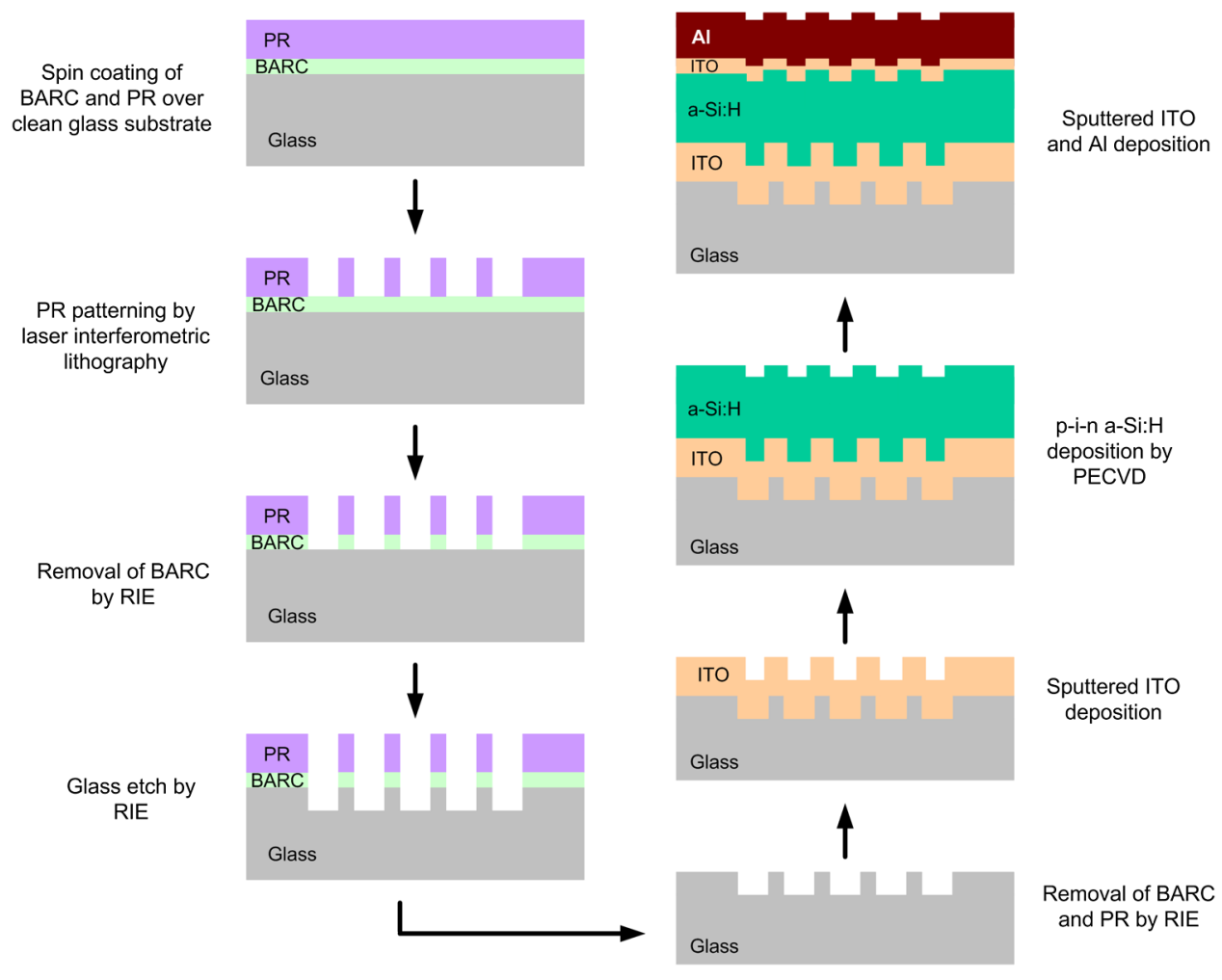

Fig. 9 Schematic view of nanopatterned solar cell fabrication steps. 


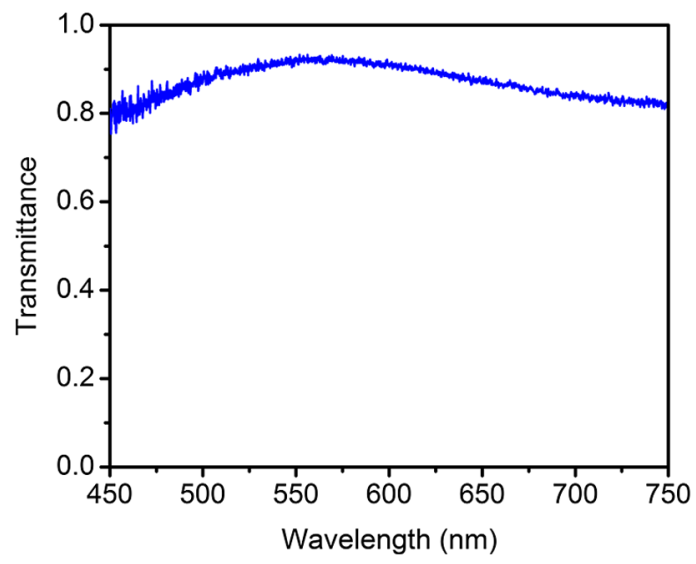

Fig. 10 Transmittance of an annealed 140-nm thick ITO film deposited on a glass substrate.

adjusted to $205^{\circ} \mathrm{C}$. A 350 -nm thick photoresist coating (PR: SEPR-701) is spin-coated at $1100 \mathrm{rpm}$ and baked for $90 \mathrm{~s}$ at $110^{\circ} \mathrm{C}$. A 1-D grating pattern with a 300-nm period is transferred into the PR by UV laser interferometric lithography using a laser with $\lambda=266 \mathrm{~nm}$. The purpose of using the BARC layer beneath the PR is to reduce the reflection from the glass substrate and ensure uniform pattern exposure over the PR. After developing the PR, the BARC is removed from the open area of the pattern by reactive ion etching (RIE) using oxygen plasma. The glass substrates are etched using an argon $(\mathrm{Ar})$ and trifluromethane $\left(\mathrm{CHF}_{3}\right)$ gas mixture. The remaining PR and BARC are removed by RIE using oxygen plasma. In a systematic experimental process, gratings with 50-, 60-, and 70-nm depths are fabricated on glass substrates. Here, we present the results obtained from solar cells with $60-\mathrm{nm}$ grating depths since the results are similar to those obtained with 50- and 70-nm grating depths.

The patterned glass substrates are coated with a 140-nm thick film of ITO by sputtering. From the scanning electron microscope (SEM) images, we confirm that ITO film deposition over the 60-nm deep grating area conforms to the grating structures and serves well as the top contact. The ITO-coated glass substrates are annealed in a rapid thermal annealer in a vacuum chamber at $490^{\circ} \mathrm{C}$ for $15 \mathrm{~min}$. The resistivity of the film is $50 \Omega / \mathrm{sq}$. The average transmittance of the annealed ITO thin film is around $90 \%$ over the 450 - to $750-\mathrm{nm}$ wavelength range. Figure 10 shows the measured transmittance of the annealed ITO film. Using a multichambered plasma-enhanced chemical vapor deposition system, a complete $\mathrm{p}-\mathrm{i}-\mathrm{n}$ single-junction solar cell with an approximate thickness of 10-nm p-type, 290-nm i-type, and 20-nm n-type a-Si:H is deposited over the ITO pattern. Finally, consecutive sputter deposition of 130-nm thick ITO and 300-nm thick aluminum films constitutes the bottom contact. Thin-film solar cells on planar
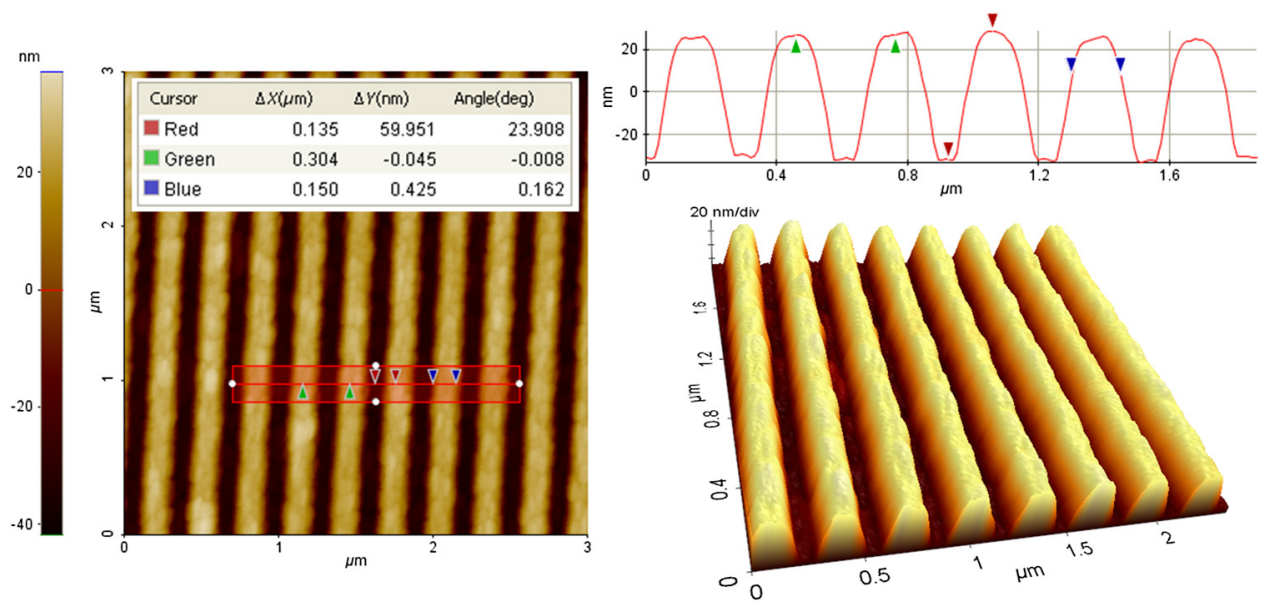

Fig. 11 Atomic force microscope surface images of an ITO-coated patterned glass substrate where $\Lambda=304 \mathrm{~nm}, d_{\mathrm{g}}=60 \mathrm{~nm}$, and $F=0.5$. 

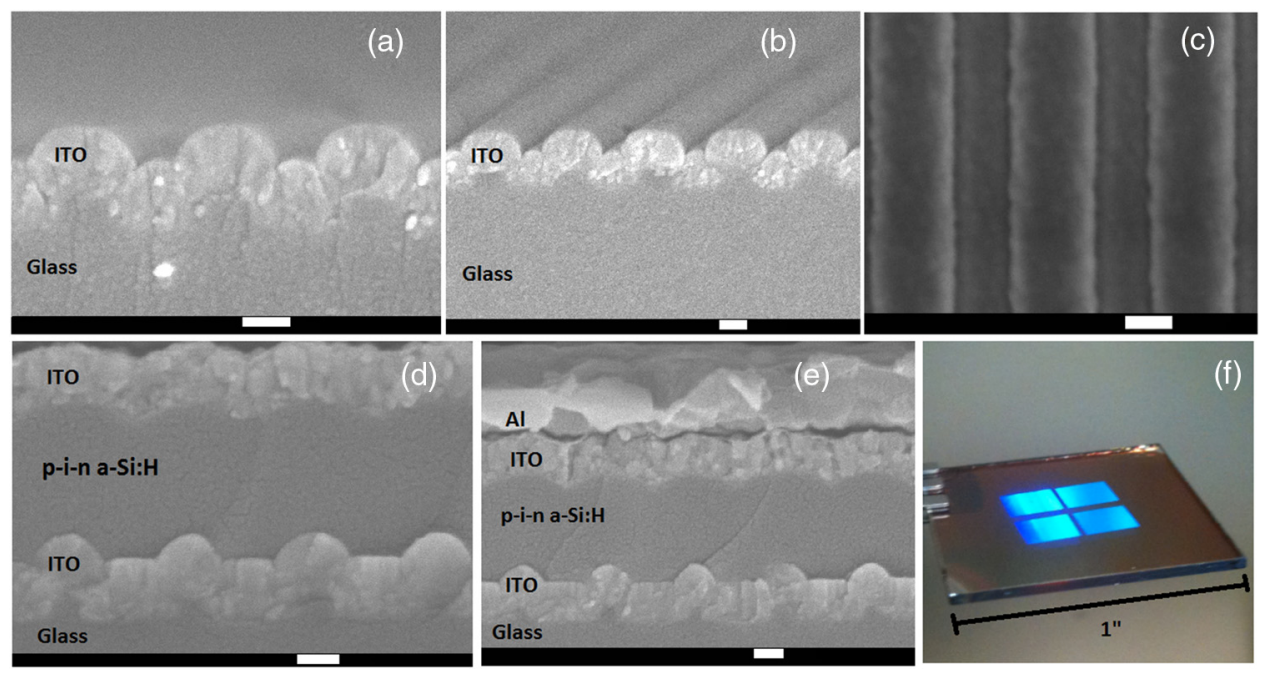

Fig. 12 (a and b) SEM cross-sectional and (c) top view of ITO-coated glass substrate; ( $d$ and e) SEM cross-sectional images of a nanopatterned solar cell; the scale bar is $100 \mathrm{~nm}$ in each image; (f) photograph of a nanopatterned solar cell.

glass substrates are also deposited with identical film thicknesses for performance comparison. Recently, we have shown that using thermal nanoimprint lithography, the nanopatterns can be fabricated by single step on plexiglas substrate which offers cost-efficient large area fabrication of nanostructured thin-film solar cells. ${ }^{32}$

The surface image of the ITO-coated oxide grating with a grating depth of $60 \mathrm{~nm}$, taken with an atomic force microscope, is shown in Fig. 11. The grating specifications are $\Lambda=304 \mathrm{~nm}$, $d_{\mathrm{g}}=60 \mathrm{~nm}$, and $F=0.5$. Figure 12 includes images from an SEM; Figs. 12(a) and 12(b) show cross-sectional images of the ITO-coated glass pattern and Fig. 12(c) shows the top view. The cross-sectional images of the complete patterned solar cell, including top and bottom contacts, are presented in Figs. 12(d) and 12(e). Figure 12(f) displays a photograph of the patterned solar cell.

\section{Results and Discussions}

\subsection{Optical Absorbance}

Regarding the superstrate structure of the solar cell, Fig. 8 illustrates that light is shone from the glass side of the structure at normal incidence. We obtain optical absorbance measurements before the bottom contacts are made in order to ascertain GMR-induced absorption enhancement exclusively. The reflected and transmitted light associated with the patterned a-Si:H solar cells are measured with a fiber optic spectrometer. The light source used is a tungsten halogen lamp with a wavelength range of 360 to $2400 \mathrm{~nm}$. We conduct the same measurements on a corresponding planar solar cell to establish a comparison. Since the grating pattern has a $300-\mathrm{nm}$ period and the refractive index of glass is 1.5 , the Rayleigh wavelength of the patterned structure is $\lambda_{\mathrm{R}}=n \Lambda=450 \mathrm{~nm}$, i.e., only the zero-order diffraction prevails beyond the $450-\mathrm{nm}$ wavelength. The primary reason for selecting a $300-\mathrm{nm}$ period is to operate the solar cells in a zeroorder diffraction regime for a substantial portion of the solar spectrum. Since no higher diffraction orders exist beyond the 450-nm wavelength, it suffices as a good approximation to measure the reflectance and transmittance for normally incident light without using an integrating sphere. We measure zero-order reflectance $\left(R_{0}\right)$ and transmittance $\left(T_{0}\right)$ spectra normalized to the source spectrum and the absorbance is obtained as $A=1-\left(R_{0}+T_{0}\right)$ for $\lambda>450 \mathrm{~nm}$. The measurement setup is schematically shown in Fig. 13.

Figure 14 shows the comparison of unpolarized absorbance between the patterned and planar solar cells. Integrated absorbance enhancement of $\sim 35 \%$ is obtained in patterned solar cells with $60-\mathrm{nm}$ deep grating depths over the wavelength range of 450 to $750 \mathrm{~nm}$. 


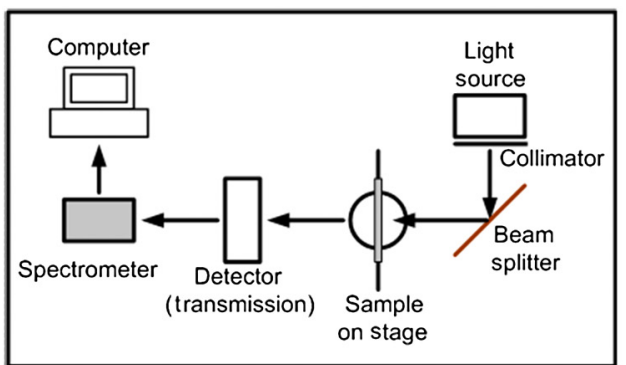

Transmission measurement

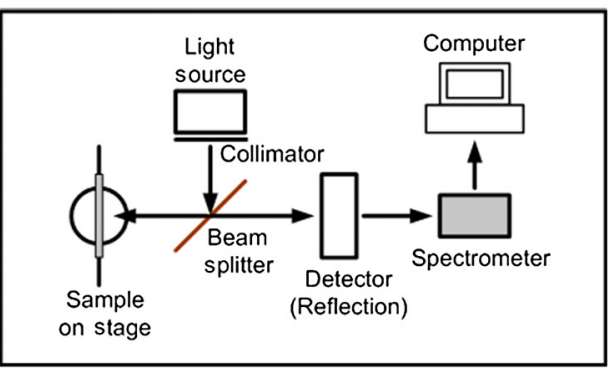

Reflection measurement

Fig. 13 Schematic view of the optical measurement setup.

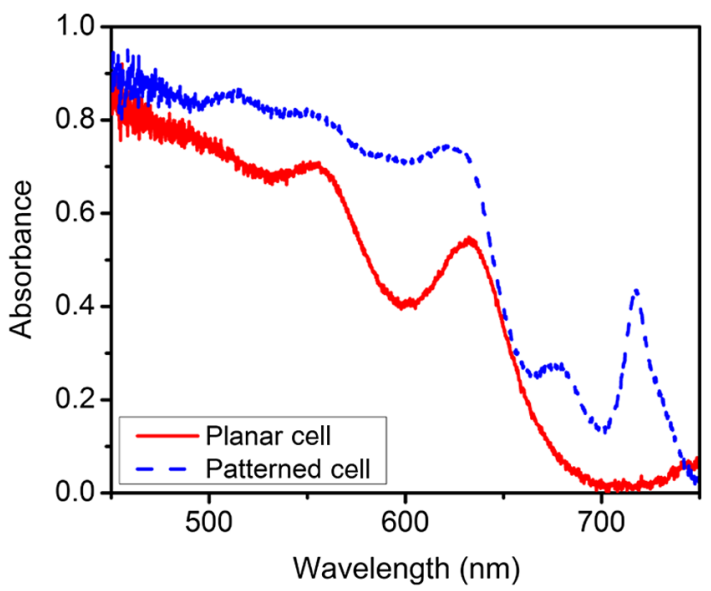

Fig. 14 Unpolarized absorbance spectra of planar and patterned solar cells at normal incidence of light.

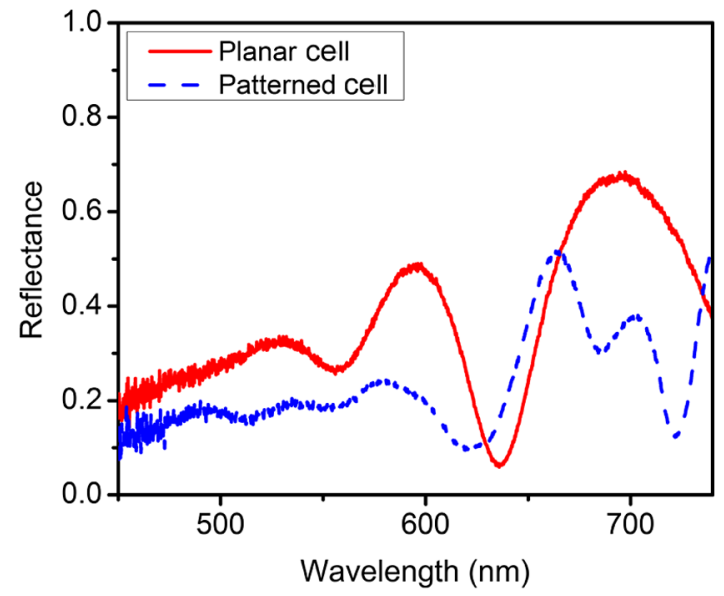

Fig. 15 Unpolarized reflectance spectra of planar and patterned solar cells at normal incidence of light.

Figure 15 shows the unpolarized reflectance comparison between planar and patterned cells. The reflectance is significantly suppressed in patterned solar cells. Designing the period to be smaller than the operating wavelength permits the antireflection effect to contribute to the enhanced optical absorbance. Due to this interdependency, it is difficult to differentiate the impact from the GMR effect. ${ }^{6}$ However, the absorption coefficient of a-Si:H weakens beyond $550 \mathrm{~nm}$. The propagation distance of the captured light along the weakly absorbing film, i.e., 


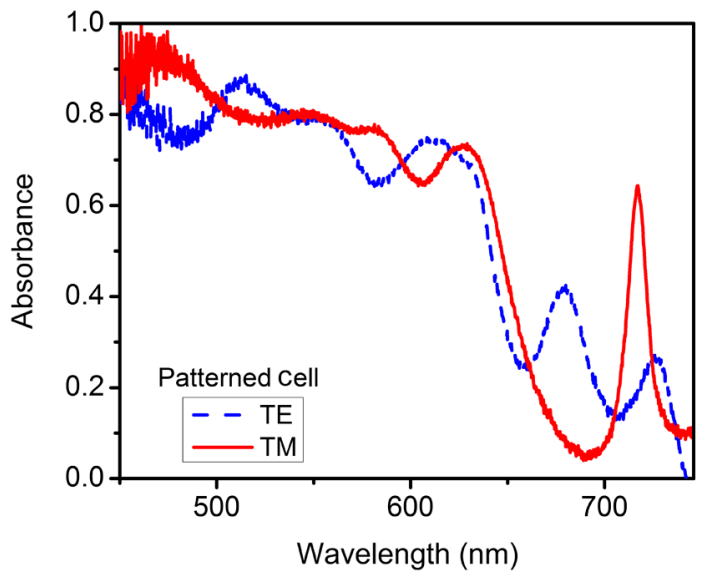

Fig. 16 TE and TM polarized components of absorbance of a patterned solar cell at normal incidence of light.

the decay length of the resonant leaky mode, must be much longer than the film thickness to achieve successful absorption in the long wavelength region. Thus, the GMR effect can ensure such optical path elongation through multiple resonances. Figure 16 shows the TE and transverse magnetic (TM) components of the total absorbance of the patterned solar cell, where the electric field vectors in TE and TM polarizations are orthogonal and parallel to the plane of incidence, respectively. As described in the previous section, the spectral signature diminishes with a higher extinction coefficient. Therefore, the individual GMR peaks are not visible over the entire wavelength range. In the long wavelength region, where the absorption coefficient is low, distinguishable GMR resonances are visible at $716 \mathrm{~nm}$ for TM polarization and at 675 and $723 \mathrm{~nm}$ for TE polarization.

The absorbance of the patterned solar cell is theoretically calculated using the RCWA computational method. The simulated and experimental absorbance comparisons for a 60-nm deep patterned solar cell are shown in Fig. 17. There are two possible reasons for the discrepancy. First, the dispersion data of a-Si:H used for simulation is not measured from the fabricated device, rather taken from literature. ${ }^{31}$ Second, the fabricated pattern does not completely follow the sharp binary pattern as schematically shown in Fig. 8. According to simulation, the integrated absorbance enhancement factor is $\sim 32 \%$ for patterned solar cell compared to a homogeneous reference, over the 450 - to $750-\mathrm{nm}$ wavelength range.

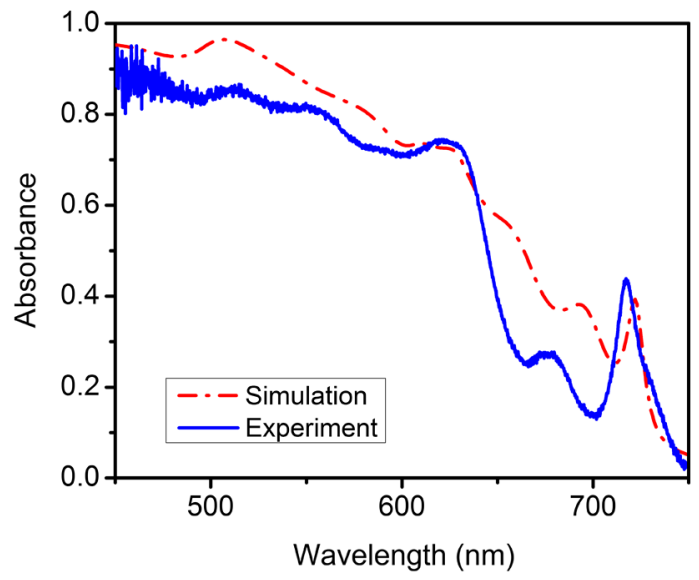

Fig. 17 Theoretical and experimental comparisons of unpolarized absorbance of patterned solar cell with $d_{\mathrm{g}}=60 \mathrm{~nm}$ at normal incidence of light. 


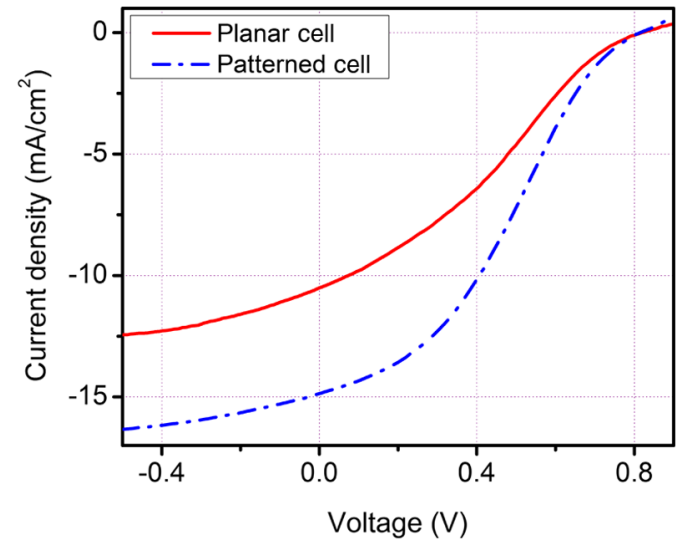

Fig. 18 Current density/voltage plot of planar and patterned solar cells.

Table 1 Electrical performance comparison.

\begin{tabular}{lccc}
\hline \hline Parameters & Planar cell & Patterned cell & Enhancement $(\%)$ \\
\hline Open-circuit voltage, $V_{\text {oc }}(\mathrm{V})$ & 0.82 & 0.82 & \\
Short-circuit current density, $J_{\mathrm{sc}}\left(\mathrm{mA} / \mathrm{cm}^{2}\right)$ & 10.5 & 14.8 & $\sim 40$ \\
Fill factor, FF $(\%)$ & 3 & 3.3 & $\sim 57$ \\
Conversion efficiency, $\eta(\%)$ & 2.6 & 4.1 & \\
\hline \hline
\end{tabular}

\subsection{Electrical Characteristics}

Solar cell efficiency measurement is carried out under AM1.5G illumination. The current density $(J)$ versus voltage $(V)$ plots for the patterned and planar solar cells are given in Fig. 18. Opencircuit voltage $\left(V_{\mathrm{oc}}\right)$, short-circuit current density $\left(J_{\mathrm{sc}}\right)$, fill factor $(\mathrm{FF})$, and conversion efficiency $(\eta)$ for the patterned and planar solar cells obtained from $J-V$ plots are summarized in Table 1.

Short-circuit current density improves by $\sim 40 \%$ from 10.5 to $14.8 \mathrm{~mA} / \mathrm{cm}^{2}$ for patterned solar cells with $d_{\mathrm{g}}=60 \mathrm{~nm}$, compared to the planar cell. Each cell has an effective area of approximately $0.1 \mathrm{~cm}^{2}$. Open-circuit voltage is $0.82 \mathrm{~V}$ for both types of solar cells. The power conversion efficiencies of the planar and patterned solar cells are measured as $2.6 \%$ and $4.1 \%$, respectively, with $\sim 57 \%$ enhancement. The fabricated solar cells exhibit rather low FFs, which is the main reason for the low conversion efficiencies obtained. FFs for the planar and patterned cells are 3 and 3.3, respectively. By enhancing the contact resistivity, a-Si:H material, and p-i-n interface quality, the FF and subsequently the efficiency of these solar cells can be improved. The higher optical absorbance obtained through GMR resonances in patterned solar cells compared to the planar reference cell are reflected in the enhanced electrical performance. The experimental results motivate the application of nanograting patterns in thin-film photovoltaics.

\section{Conclusion}

We explain light trapping in thin-film solar cells through GMRs with numerical examples and experimentally implement nanograting patterns into a-Si:H solar cells with $300-n m$ periods and 60-nm grating depths. Theoretically simulated GMR-enabled enhanced optical absorption is experimentally observed in fabricated patterned solar cells, which leads to improved electrical performance. For the particular device design used, integrated absorbance enhancement of $\sim 35 \%$ over the 450 - to 750 -nm wavelength range and short-circuit current density enhancement of $\sim 40 \%$ are found in a $60-\mathrm{nm}$ deep nanograting patterned solar cell compared to an equivalent 
planar reference cell. GMR phenomena strongly depend on dispersion properties and thickness of the absorbing photovoltaic materials. Enhanced optical absorbance leading to an increased conversion efficiency holds the same potential for micro-crystalline and organic thin-film solar cells with optimal structural and material device parameters.

\section{Acknowledgments}

The authors thank MVSystems, Inc. for a-Si:H deposition and Wenhua Wu for computational assistance. Partial support was provided by the UT System Texas Nanoelectronics Research Superiority Award funded by the State of Texas Emerging Technology Fund as well as by the Texas Instruments Distinguished University Chair in Nanoelectronics Endowment.

\section{References}

1. M. A. Green, "Thin-film solar cells: review of materials, technologies and commercial status," J. Mater. Sci: Mater. Electron. 18(S1), S15-S19 (2007), http://dx.doi.org/10.1007/ s10854-007-9177-9.

2. A.V. Shah et al., "Thin-film silicon solar cell technology," Prog. Photovolt. 12(2-3), 113142 (2004), http://dx.doi.org/10.1002/(ISSN)1099-159X.

3. S. B. Mallick et al., "Coherent light trapping in thin-film photovoltaics," MRS Bull. 36(6), 453-460 (2011), http://dx.doi.org/10.1557/mrs.2011.113.

4. C. Heine and R. H. Morf, "Submicrometer gratings for solar energy applications," Appl. Opt. 34(14), 2476-2482 (1995), http://dx.doi.org/10.1364/AO.34.002476.

5. N.-N. Feng et al., "Design of highly efficient light-trapping structures for thin-film crystalline silicon solar cells," IEEE Trans. Electron Devices 54(8), 1926-1933 (2007), http://dx .doi.org/10.1109/TED.2007.900976.

6. M. Kroll et al., "Employing dielectric diffractive structures in solar cells-a numerical study," Phys. State Solidi A 205(12), 2777-2795 (2008), http://dx.doi.org/10.1002/pssa.v205:12.

7. J. Muller et al., "TCO and light trapping in silicon thin-film solar cells," Sol. Energy 77(6), 917-930 (2004), http://dx.doi.org/10.1016/j.solener.2004.03.015.

8. K.-C. Lai et al., "Texturing of the back reflector for light trapping enhancement in micromorph thin film solar cells," Thin Solid Films 519(11), 3946-3949 (2011), http://dx.doi.org/ 10.1016/j.tsf.2011.01.360.

9. S.-S. Lo et al., "Broad-band anti-reflection coupler for a:Si thin-film solar cell," J. Phys. D: Appl. Phys. 40(3), 754-758 (2007), http://dx.doi.org/10.1088/0022-3727/40/3/010.

10. Y. M. Song, J. S. Yu, and Y. T. Lee, "Antireflective submicrometer gratings on thin-film silicon solar cells for light-absorption enhancement," Opt. Lett. 35(3), 276-278 (2010), http://dx.doi.org/10.1364/OL.35.000276.

11. H. A. Atwater and A. Polman, "Plasmonics for improved photovoltaic devices," Nat. Mater. 9(3), 205-213 (2010), http://dx.doi.org/10.1038/nmat2629.

12. H.-Y. Lin et al., "Surface plasmon effects in the absorption enhancements of amorphous silicon solar cells with periodical metal nanowall and nanopillar structures," Opt. Express 20(S1), 104-118 (2012), http://dx.doi.org/10.1364/OE.20.00A104.

13. S. Y. Chou and W. Ding, "Ultrathin, high-efficiency, broad-band, omni-acceptance, organic solar cells enhanced by plasmonic cavity with subwavelength hole array," Opt. Express 21(S1), A60-A76 (2013), http://dx.doi.org/10.1364/OE.21.000A60.

14. J. G. Mutitu et al., "Thin film silicon solar cell design based on photonic crystal and diffractive grating structures," Opt. Express 16(19), 15238-15248 (2008), http://dx.doi.org/10 $.1364 / \mathrm{OE} .16 .015238$.

15. L. Zeng et al., "Demonstration of enhanced absorption in thin-film Si solar cells with textured photonic crystal back reflector," Appl. Phys. Lett. 93(22), 221105 (2008), http://dx.doi .org/10.1063/1.3039787.

16. B. Curtin, R. Biswas, and V. Dalal, "Photonic crystal based back reflectors for light management and enhanced absorption in amorphous silicon solar cells," Appl. Phys. Lett. 95(23), 231102 (2009), http://dx.doi.org/10.1063/1.3269593. 
17. Y.-C. Lee et al., "Enhanced light trapping based on guided mode resonance effect for thinfilm solar cells with two filling-factor gratings," Opt. Express 16(11), $7969-7975$ (2008), http://dx.doi.org/10.1364/OE.16.007969.

18. K. Söderström et al., "Photocurrent increase in n-i-p thin-film silicon solar cells by guided mode excitation via grating coupler," Appl. Phys. Lett. 96(21), 213508 (2010), http://dx.doi .org/10.1063/1.3435481.

19. E. Garnett and P. Yang, "Light trapping in silicon nanowire solar cells," Nano Lett. 10(3), 1082-1087 (2010), http://dx.doi.org/10.1021/nl100161z.

20. J. Zhu et al., "Nanodome solar cells with efficient light management and self-cleaning," Nano Lett. 10(6), 1979-1984 (2010), http://dx.doi.org/10.1021/n19034237.

21. J. Kim et al., "Three-dimensional a-Si:H solar cells on glass nanocone arrays patterned by self-assembled Sn nanospheres," ACS Nano 6(1), 265-271 (2012), http://dx.doi.org/10 $.1021 / \mathrm{nn} 203536 x$.

22. R. Magnusson and S. S. Wang, "New principle for optical filters," Appl. Phys. Lett. 61(9), 1022-1024 (1992), http://dx.doi.org/10.1063/1.107703.

23. S. S. Wang and R. Magnusson, "Theory and applications of guided-mode resonance filters," Appl. Opt. 32(14), 2606-2613 (1993), http://dx.doi.org/10.1364/AO.32.002606.

24. D. Shin et al., "Thin-film optical filters with diffractive elements and waveguides," Opt. Eng. 37(9), 2634-2646 (1998), http://dx.doi.org/10.1117/1.601764.

25. D. Rosenblatt, A. Sharon, and A. A. Friesem, "Resonant grating waveguide structures," IEEE J. Quantum Electron. 33(11), 2038-2059 (1997), http://dx.doi.org/10.1109/3.641320.

26. Y. Ding and R. Magnusson, "Resonant leaky-mode spectral-band engineering and device applications," Opt. Express 12(23), 5661-5674 (2004), http://dx.doi.org/10.1364/OPEX.12 .005661 .

27. K. J. Lee et al., "Resonant wideband polarizer with single silicon layer," Appl. Phys. Lett. 98(21), 211112 (2011), http://dx.doi.org/10.1063/1.3594244.

28. M. J. Uddin and R. Magnusson, "Highly efficient color filter array using resonant $\mathrm{Si}_{3} \mathrm{~N}_{4}$ gratings," Opt. Express 21(10), 12495-12506 (2013), http://dx.doi.org/10.1364/OE.21 .012495 .

29. T. K. Gaylord and M. G. Moharam, "Analysis and applications of optical diffraction by gratings," Proc. IEEE 73(5), 894-937 (1985), http://dx.doi.org/10.1109/PROC.1985.13220.

30. M. Faryad and A. Lakhtakia, "Enhancement of light absorption efficiency of amorphoussilicon thin-film tandem solar cell due to multiple surface-plasmon-polariton waves in the near-infrared spectral regime," Opt. Eng. 52(8), 087106 (2013), http://dx.doi.org/10.1117/1 .OE.52.8.087106.

31. A. Fontcuberta i Morral and P. Roca i Cabarrocas, "Structure and hydrogen content of polymorphous silicon thin films studied by spectroscopic ellipsometry and nuclear measurements," Phys. Rev. B 69(12), 125307 (2004), http://dx.doi.org/10.1103/PhysRevB.69 .125307.

32. T. Khaleque, H. Svavarsson, and R. Magnusson, "Fabrication of resonant patterns using thermal nano-imprint lithography for thin-film photovoltaic applications," Opt. Express 21(S4), A631-A641 (2013), http://dx.doi.org/10.1364/OE.21.00A631.

Tanzina Khaleque is a graduate research assistant in the Department of Electrical Engineering at the University of Texas-Arlington. She received her BS and MS degrees in electrical and electronic engineering from Bangladesh University of Engineering and Technology in 2006 and 2009, respectively. She is pursuing her PhD degree in electrical engineering at UT-Arlington.

Robert Magnusson is a professor of electrical engineering and the Texas Instruments Distinguished University Chair in Nanoelectronics at the University of Texas-Arlington. He received his $\mathrm{PhD}$ degree from the Georgia Institute of Technology. He is the author of $\sim 400$ journal and conference papers. Current research interests include periodic nanostructures, nanolithography, nanophotonics, nanoelectronics, nanoplasmonics, and optical bio- and chemical sensors. He is a fellow of SPIE, OSA, IEEE, and the National Academy of Inventors. 